\title{
INSTRUMENTO PARA LA CARACTERIZACIÓN ELÉCTRICA DE GENERADORES FOTOVOLTAICOS
}

Dr.Firman, Andrés D. ${ }^{1}$; Dr. Vera, Luis H. ${ }^{1}$; Dr. Toranzos, Víctor J. ${ }^{1}$; Dr. Cáceres, Manuel $^{1}$; Dr. Busso, Arturo J. ${ }^{1}$

Palabrasclave:Energía Solarfotovoltaica, instrumentación, caracterización eléctrica.

Resumen: El presente trabajo, trata sobre el diseño y la fabricación de un instrumento destinado a la caracterización eléctrica de generadores fotovoltaicos. Dicha caracterización cobra importancia debido a que permite inferir el estado de funcionamiento del generador, realizardiagnósticos o realizar estimaciones de la producción energética, entre otros. La caracterización mencionada se realiza mediante la adquisición de la curva I-V (corriente-tensión) del generador fotovoltaico. El Grupo en Energías Renovables G.E.R. de la UNNE, ha desarrollado un trazador de curvas I-V que funciona por un método innovador de polarización del arreglo fotovoltaico, denominado híbrido. La metodología se basa en la combinación de las tradicionales cargas de tipo activa y capacitiva (aprovechando las ventajas asociadas a cada uno). El instrumento permite determinar pro- blemas de generación en instalaciones fotovoltaicas rurales ubicadas en la región. Suprincipio de funcionamientocuenta actualmente con una patente Nacional.

\section{Introducción}

En los generadores fotovoltaicos es importante conocer en detalle la cantidad de energía que se puede obtener, el rendimiento del sistema, los posibles problemas que puedan ocurrir en condiciones normales de funcionamiento $y$, de este modo, verificar las condiciones de operación de este tipo de instalaciones. Por lo que contar con información confiable al respecto de las características eléctricas de los dispositivos fotovoltaicos $(\mathrm{FV})$ resulta transcendente tanto para fabricantes como para proyectistas. Para los fabricantes, permite la clasificación de los módulos, verificación de fallas y la producción de documentación técnica de alta calidad. Para los proyectistas de sistemas fotovoltaicos, el aumento

\footnotetext{
${ }^{1}$ Grupo en Energías Renovables, Facultad de Ciencias Exactas y Naturales y Agrimensura. UNNE. Av. Libertad 5460. Corrientes.

Dr. Andrés D. Firman. 0379-154400227. afirman@ger-unne.com.ar

Dr. Luís H. Vera.0362-154660430.1vera@ger-unne.com.ar

Dr. Víctor J. Toranzos. 0379-154661747.vtoranzos@ger-unne.com.ar

Dr. Manuel Cáceres.0379-154268000. mcaceres@ger-unne.com.ar

Dr. Arturo J. Busso.0379-154670183. ajbusso@ger-unne.com.ar
} 
de confiabilidad en las especificaciones de los módulos fotovoltaicos permite la optimización en el diseño, una determinación precisa de la capacidad de generación, lo que lleva a un cálculo preciso del tiempo de retorno de la inversión.En casos de análisis de plantas generadoras $\mathrm{FV}$, posee utilidad para actuar en casos de auditorías.

Como es de esperar para sistemas construidos a base de dispositivos semiconductores, los componentes de un generador fotovoltaico, es decir, los módulos y en estos las celdas FV, se caracterizan eléctricamente a través del trazado de su respuesta I-V, realizada para ciertas condiciones definidas (IRAM, 1998). Dicho trazado, se conoce como curva corrientevs tensión (curvaI-V).

Para obtener la curva I-V a sol natural, deben medirse pares de valores de corrientes y tensiones en intervalos regulares de tiempo, normalmente este proceso se realiza durante lapsos menores a los 100 ms. Este ensayo se realiza por medio de la desconexión física de los generadores fotovoltaicos del resto del sistema y posteriormente se conecta a ellos una carga variable. Al trabajar con la curva mencionada, posibilita ajustar modelos que ayudan a explicar o anticipar el comportamiento del sistema descrito por la Ec. 1, (Kichou et al, 2016, Barth et al, 2016, Jordehi, 2016). Entre los parámetros más representativos de esta curva, están la corriente de corto circuito $(I c c)$, la tensión de circuito abierto $(V c a)$ y el punto de potencia máxima $(P m)$.

La Ec. 1 describe la curva I-V mencionada, en esta; $m$ es el índice de idealidad del diodo, $R s$ su resistencia serie, $R p$ la resistencia paralelo, $N$ es el número de celdas conectadas eléctricamente en serie y $V t$ el potencial térmico que depende de la temperatura de celda FV (Firman et al. 2013).

$$
I=I c c\left(1-\exp \left(\frac{V-V c a+I \cdot R s}{m N V t}\right)\right)-\frac{V+I \cdot R s}{R p}
$$

(Ec. 1)

La necesidad de diagnosticar sistemas fotovoltaicos autónomos distribuidos en la región, impulsó el diseño e implementaciónde un instrumento portátil capaz de encargarse de la tarea, sumado al hecho que en el mercado nacional actual no es común encontrarlos y $\mathrm{mu}^{-}$ chas veces su costo resulta prohibitivo.

Los equipos dedicados al trazado de curvas I-V de dispositivos FV del mercado, funcionan de dos formas principales; controlando una carga electrónica o bien, de forma analógica, monitoreando el transitorio de carga de un banco de capacitores, de capacidad suficiente para lograr el tiempo de barrido requerido (Kuai y Yuvarajan, 2006, Muñoz y Lorenzo, 2005).

El instrumento desarrollado presenta una innovación en este sentido, la cual consiste en un tipo nuevo de carga variable, llamada "carga hibrida", la cual utiliza los dos métodos tradicionalmente empleados. El funcionamiento se basa en una combinación entre las cargas capacitivas y cargas electrónicas convencionales, imitando a un banco de capacitores, de esta forma, a través del control de un capacitor conectado en la base de un transistor bipolar, se realiza el barrido de tensión de un dispositivo fotovoltaico, desde el estado de corto circuito al de circuito abierto. Este capacitor se carga con la corriente de base $\beta$ veces menor que la corriente de colector por la que circula la del dispositivo $\mathrm{FV}$, siendo $\beta$ la ganancia en corriente del transistor bipolar. Normalmente, para transistores de potencia $\beta$ se encuentra en el orden de 50 a 100 (Millman y Halkias, 1972). 
Las ventajas de la combinación sobre las cargas tradicionales son; reducción del tamaño del banco de capacitores para lograr tiempos de barrido similares y la simplicidad de manejo y control en comparación con las cargas electrónicas activas. Una reseña electrónicamente más detallada puede encontrarse en la publicación realizada en la Asociación Argentina de Energías Renovables y Ambiente, Firman et al, 2010.

\section{Contraste del instrumento desarrolla- do}

El prototipo desarrollado se contrastó en el laboratorio de Universidad Federal de Rio grande do Sul (UFRGS) en Porto Alegre (Brasil) con un instrumento confiable y ampliamente probado, el cual consta de una fuente de tensión variable $\mathrm{KEPCO}$ modelo BOP 100-10 MG y dos multímetros Agilent Technologies modelo 3458A de
6 dígitos y medio para la adquisición de tensión y corriente. Utilizando el sistema de la UFRGS y el prototipo a prueba, se tomaron simultáneamente curvas $\mathrm{I}-\mathrm{V}$ para un grupo de módulos $\mathrm{FV}$ de $50 \mathrm{Wp}$. El contraste de ambos equipos indica que el prototipo desarrollado posee desvíos menores al 2,0 \% en el punto de máxima potencia, lo que se considera aceptable para el caso de un instrumento portátil de campo.

\section{Descripción del trazador de curvas I-V}

El equipo desarrollado (Figura 1a) consta de un gabinete principal, diseñado en forma de maletín metálico, conveniente para su traslado y protección. Se aprecia en el instrumento su característica portable ya que está diseñado para ser trasladado hasta donde se encuentran las instalaciones FV autónomas, estas muchas veces, ubicadas en lugares de difícil acceso de nuestra región.

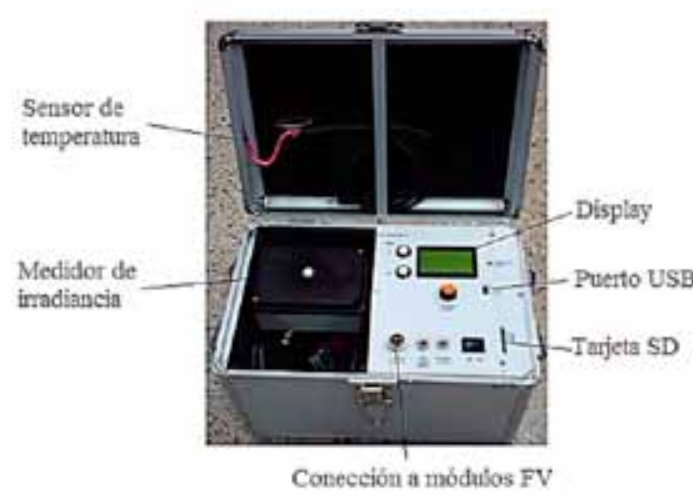

(a)

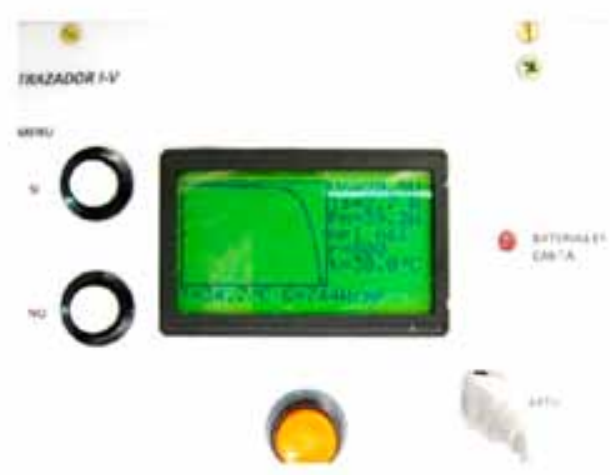

(b)

Figura 1: (a) Equipo trazador de curvas I-V hibrido, componentes principales. (b) Panel del instrumento.

Como componentes principales presenta un "sensor de temperatura" el cual va conectado al "medidor de irradiancia". Este medidor de irradiancia es de características inalámbricas, por lo que transmite los valores de irradiancia y temperatura medida hacia el módulo principal, facilitando su instalación. El instrumento posee un "display" gráfico de $128 \times 64 \mathrm{px}$ donde se presenta la información y se grafica la curva I-V adquirida. El display también muestra la información pertinente a las variables ambientales en el 
momento del ensayo, visualiza la tensión de circuito abierto, la corriente de corto circuito y la potencia máxima en esas condiciones (Figura 1b). También resulta útil para navegar por las diferentes opciones del menú.

Los datos registrados pueden ser almacenados en forma de archivo de texto plano, en la tarjeta de memoria tipo SD. El registro en tarjeta SD permite que la información sea fácilmente extraída para su análisis y traslación a condiciones estándar de medida. La tarea de extracción, también puede realizarse automáticamente por transmisión a PC por puerto USB y un software específicamente desarrollado.

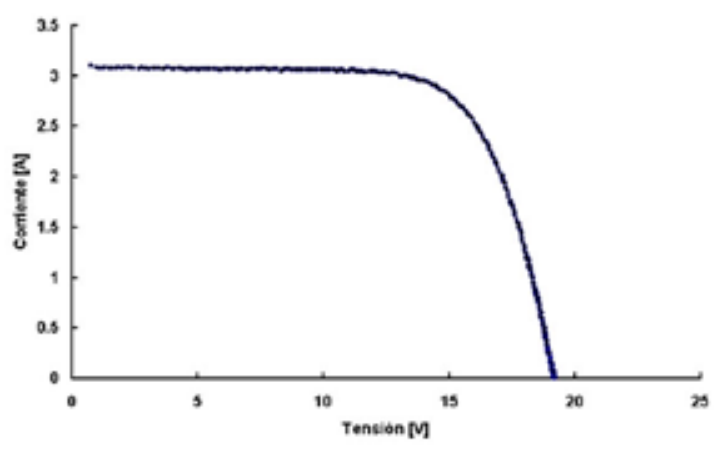

(a)

\section{Resultados obtenidos.}

El uso del instrumento trazador de curvas I-V en la práctica, resulta en la obtención de curvas I-V donde se verificaron módulos y generadores $\mathrm{FV}$ de silicio policristalino de distintas potencias y estados de funcionamiento. La Figura 2a, muestra la curva I-V, de un dispositivo $\mathrm{FV}$ de $50 \mathrm{Wp}$, que en base a su perfil y a su estudio mediante traslación a condiciones estándar de medida, realizada con un software desarrollado para ese fin, se logra determinar que este módulo funciona correctamente. Se aprecia además en esta, el perfil típico de la curva I-V.

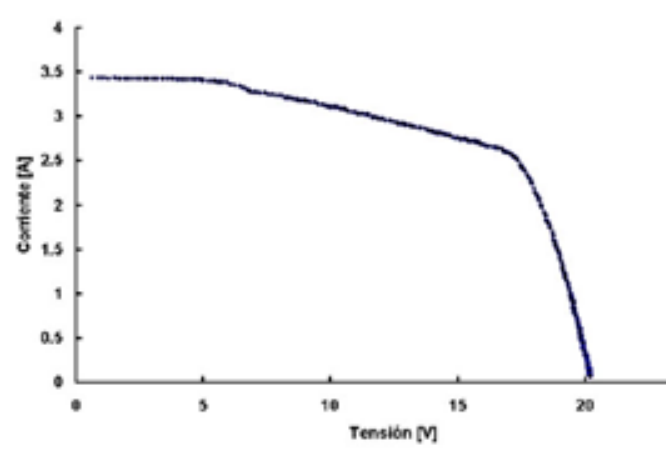

(b)

Figura 2: (a) Curva I-V de un módulo de $50 \mathrm{Wp}$ de correcto funcionamiento. (b) Curva I-V de un módulo de $50 \mathrm{Wp}$, que presenta defectos debido a posible defecto en sus celdas componentes.

La Figura 2b, muestra la curva de un módulo de $50 \mathrm{Wp}$, en la que en base a las mediciones realizadas con el trazador desarrollado y la observación de su perfil, se puede apreciar un defecto, que se supone que puede deberse a posible malfuncionamiento en una de sus celdas componentes semiconductoras, lo que ocasiona una pérdida de potencia sensible, afectando la potencia máxima que este módulo es capaz de entregar. Este estudio fue llevado a cabo por medio del análisis cualitativo del mo- delo equivalente eléctrico de las 36 celdas que componen el módulo FV, la cual se encuentra publicada en Firman et al, 2013.

La Figura 3a, muestra la curva obtenida de un generador FV de $200 \mathrm{Wp}$ donde se aprecian defectos de acoplamiento entre los módulos que lo componen (mismatchlosses), lo que produce en consecuencia una caída en la zona plana de la curva afectando a la potencia máxima que puede obtenerse de este. 
En todos los casos la metodología de traslación fue realizada por empleo de la meto- dología propia, la que se encuentra registrada en la publicación de Montes et al, 2016.

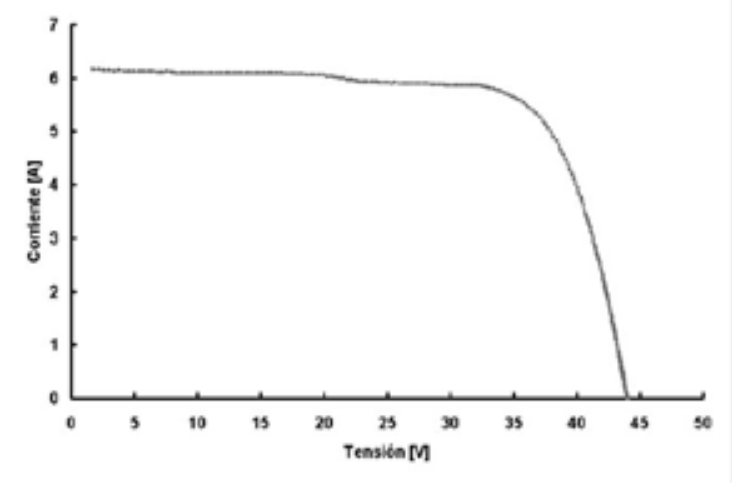

(a)

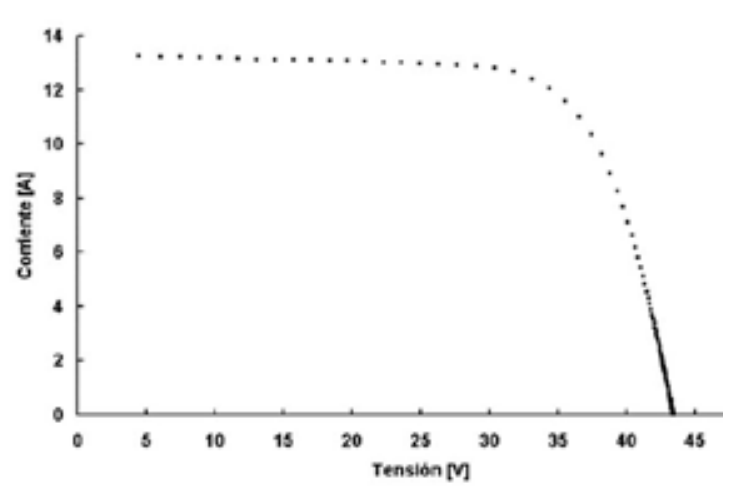

(b)

Figura 3: (a) Curva I-V de un arreglo fotovoltaico de 200Wp con defectos de acoplamiento. (b) Curva I-V de un arreglo fotovoltaico de $400 \mathrm{Wp}$ sin presentar defectos.

Por último la Figura 3b, muestra una curva I-V de un arreglo de 400 Wp logra$\mathrm{da}$ con el instrumento diseñado (potencia máxima admisible por el instrumento), de la que se comprueba, en base a la observación del perfil de la curva y posterior traslación, condiciones óptimas de funcionamiento.

\section{Conclusiones}

El Instrumento Trazador de curvas I-V para caracterización eléctrica de módulos fotovoltaicos basado en el sistema híbrido desarrollado permite caracterizar eléctricamente generadores fotovoltaicos de hasta $400 \mathrm{Wp}$ utilizando un método innovador que combina las ventajas presentadas por los sistemas de polarización con carga activa y con carga capacitiva. Proporciona una alternativade bajo ruido, reducido volumen y de bajo costo, por medio de la cual se obtienen un suficiente número de puntos en el trazado de la curva I-V de generadores fotovoltaicos, lo que permite un ajuste aceptable. Ofrece una característica eléctrica similar a la obtenida con un banco de capacitores con solamente un capacitor cuyo valor es de 50 a 100 veces menor a la de una carga capacitiva de igual tamaño.

Mediante el empleo de un dispositivo trazador basado en la carga hibrida descripta, se pueden obtener curvas I-V de calidad, por lo que se constituye en una herramienta útil para la verificación y detección de fallas en los generadores de instalaciones fotovoltaicas autónomas.

El instrumento desarrollado y su metodología de funcionamiento cuentan actualmentecon una patente Nacional. 


\section{Bibliografía}

Barth, N., Jovanovic, R., Ahzi, S., \&Khaleel, M. A. (2016). PV panel single and doublediodemodels: Optimization of theparameters and temperaturedependence. Solar EnergyMaterials and Solar Cells, 148, 87-98.

Busso, J. Firman, A.Toranzos, V. Cáceres, M. Vera L. (2011). Sistema híbrido para la caracterización eléctrica de arreglos fotovoltaicos. Acta INPI $\mathrm{N}^{\circ}$ 20110104031. Nota SGRI 180/14 Universidad Nacional del Nordeste. Argentina.

Firman, A. Toranzos, V. Busso, A. Cadena, C. Vera, L. (2010). Sistema híbrido para la caracterización eléctrica de arreglos fotovoltaicos. Avances en energías renovables y medio ambiente. Asociación Argentina de Energía Solar. ISBN/ ISSN: 0329-5184.

Firman A., Toranzos, V. Busso, A. Vera, L. de la Casa J. (2013). Qualitativeanalysis of electricalmismatchlosses in photovoltaicdevices. EUPVSEC, Congreso Europeo de fotovoltaica, París Francia. ISBN: 3-936338-33-7. pp 3212 - 3215.

IRAM (1998) Norma IRAM 210013-2. Módulos fotovoltaicos. Características eléctricas en condiciones normalizadas.
Kichou, S., Silvestre, S., Guglielminotti, L., Mora-López, L., \& Muñoz-Cerón, E. (2016). Comparison of two PV arraymodelsforthesimulation of PV systemsusingfivedifferentalgorithmsfortheparametersidentification. RenewableEnergy, 99, 270-279.

Jordehi, A. R. (2016). Parameterestimation of solar photovoltaic (PV) cells: A review. Renewable and SustainableEnergyReviews, 61, 354-371.

Kuai Y. y Yuvarajan S. (2006). An electronic load for testing photovoltaic panels. Journal of Power Sources 154. pp. 308-313.

Millman J. y Halkias C. (1972) Analog and digital circuits and systems. McGraw-Hill KogakushaTokyopp 911.

Muñoz J. y Lorenzo E. (2005) Diseño de una carga capacitiva para medir generadores fotovoltaicos. Era solar: Energías renovables, ISSN 0212-4157, No. 127, pp. 38-49.

Montes-Romero, J., Torres-Ramírez, M., de la Casa, J., Firman, A., \& Cáceres, M. (2016, June). Software toolfortheextrapolation to Standard Test Conditions (STC) from experimental curves of photovoltaic modules. In 2016 Technologies Applied to Electronics Teaching (TAEE) (pp. 1-7). IEEE. 